M. Grahl, J. Svensson, A. Werner, T. Andreeva, S. Bozhenkov, M. Drevlak, J. Geiger,

M. Krychowiak, Y. Turkin and the W7-X Team

\title{
Web Services for 3D MHD Equilibrium Data at Wendelstein 7-X
}

\begin{abstract}
Consistent physics and engineering models are required for many use cases at Wendelstein 7-X. Web service technology is used to provide convenient access to common software components and experiment data. The usage of standard web protocols allows the integration in client software written in any programming language. This leads to a better reusability of expert codes and experiment data by a large international team. At W7-X, web services provide common functions such as Biot-Savart calculations and magnetic field line tracing. Further services provide access to databases for diagnostic data or machine models. A new web service was implemented for the creation of 3D magnetohydrodynamic (MHD) equilibrium calculations and a user-friendly access to the results. Consistent interface definitions allow an orchestration of the W7-X web services by user code, as well as a seamless integration into a data analysis framework. In this paper, we present the current state of the MHD equilibrium web services at Wendelstein 7-X.
\end{abstract}

\section{Introduction}

The optimized stellarator Wendelstein 7-X (W7-X) started its operation in December 2015 [1]. W7-X research aims for good plasma confinement and the demonstration of steady-state operation. This could make the stellarator a serious option for a future fusion power plant. Magnetohydrodynamic (MHD) equilibrium model information is needed at W7-X for many use cases [2], including diagnostic design and data analysis [3] or plasma operation (e.g. on-/off-axis heating scenarios). Due to the geometry of stellarators a three-dimensional MHD equilibrium model is needed. This leads to codes which are much more complex and time consuming than $2 \mathrm{D}$ equilibrium reconstruction for axisymmetric configurations. For example the widely-used 3D equilibrium code VMEC [4] takes minutes to hours for such computations. Therefore the VMEC equilibrium data for $\mathrm{W} 7-\mathrm{X}$ configurations has to be calculated in advance and stored in a database.

Web service technology is used to provide convenient access to equilibrium model data for the whole W7-X team. Standard protocols allow the integration of complex models in any client software and the combination of expert code on an application level. At Wendelstein 7-X a growing number of web services are implemented and in use. For example, the W7-X Archive Web API provides unified access to the data of all diagnostic systems and machine operation data. Further examples are web services for the calculation of 3D magnetic fields, magnetic field line tracing, as well as web service interfaces for a coil geometry database and a database for 3D meshes of machine parts and components. A VMEC web service allows the integration of MHD equilibrium calculations. In addition a surrogate model service is available, which can provide an approximation of equilibrium information in milliseconds. Unified interfaces with matching data types allow orchestration of the W7-X web services: results can be used directly as input for other services. Expert codes and model data can be recombined for other use cases on an application level. In this way the web service approach leads to a better interoperability of codes and also increases the traceability of calculation results. Most of the services use generic data models and can therefore be used for calculations for other machines as well.

\section{Web Services and SOA}

The requirements for a software integration framework suitable for stellarator research were shown in [5], suggesting a solution based on service oriented architecture (SOA) using web services [3], [6]. 
Web services provide a straightforward way to integrate distributed software components into all kinds of user software over a local network or the internet. The usage of open standards leads to interoperability: server- and client part do not have to be implemented in the same programming language. This increases significantly the reusability of codes provided by the domain experts. Software components can be accessed by a standard web interface and therefore be easily used; even from remote. Experiment data and complex physics models can be integrated in software written in a preferred programming language: C, Java, Python, MATLAB, LabView, or IDL. On the server-side, the service implementation can be easily moved from development on a PC into production stage on a server, which also includes cluster and Cloud-based environments. By adding more service endpoints, a service can be scaled horizontally to meet the user demand.

\section{Web Service Standards}

A large number of web service techniques were introduced by the industry over the last decades. The most successful ones allow an interoperable communication of client and server by using standard protocols for message encoding and transport. Text-based standards, like JavaScript Object Notation (JSON) and Extensible Markup Language (XML), are often used for message encoding, while the Hypertext Transfer Protocol (HTTP) is used in most cases for message transport. As fusion experiments often have long operations times of twenty years and more, well established technologies and standards are preferred. For these reasons the widespread SOAP and Representational State Transfer (REST) are used for the implementation of web services at W7-X.

SOAP (once an acronym for Simple Object Access Protocol) and Web Services Definition Language (WSDL) are XML-based standards, which are used for many years in industry and science. Therefore libraries which support SOAP/WSDL web services are implemented in most programming languages. For using a SOAP/WSDL web service, the client software generates stub-classes from the interfaces description provided by a WSDL file. These classes contain methods named as the web service functions, but with input and output parameters using the data types of the target platform. When the user calls these local methods the server communication, including transformation to SOAP messages, is handled automatically by the client library.

REST is an architecture style which is used for many services on the internet. In a REST API, different resources on a server are accessible via a specific web address. Typically only HTTP operations (e.g. GET and POST) are used to access representations of the resources. In many REST APIs the resources can requested in different formats. For example websites are often used for human interaction, while JSON is used for a machine-readable representation of the data. JSON is an easy to parse format which is also very compact: message payloads are up to four times smaller than in SOAP/XML.

\section{SOA Infrastructure}

In service oriented architecture (SOA) solutions multiple web services are available providing selfcontained functions. These services can be (re-)combined for different use cases. For the operation of a large SOA a suitable infrastructure is needed.

At Max-Planck-Institut für Plasmaphysik (IPP) Greifswald, a private Cloud based on VMware vSphere [9] is used for hosting service endpoints on virtual machines (Fig. 1). This allows a flexible management of computing resources based on the current user demand. The individual services can be adjusted in number of servers, CPUs, and memory size. Next to the hosting of service instances, some additional features were established. For example a SOA website acts as a central place for information about W7$\mathrm{X}$ web services on the institute intranet. This includes starter guides in different programming languages and documentation of service functions, links to the WSDL interface descriptions, and sample codes. The websites also provides interactive tools, written in JavaScript, for online testing of many web service functions (see Fig. 2 and Fig. 3). For example database queries, 2D flux surface plots, and also 3D visualization of model data. Several of the W7-X web services provide functions for status monitoring for long running calculations. The status information is also shown on the website. 
An ESB (Enterprise Service Bus) is used as a central component to distribute the load of service requests to different service endpoints. ESB products provided many functions for different business integration scenarios, but only a few are suitable for a scientific use case. At the moment endpoint abstraction via proxy services are used for load-balancing and interface versioning. Other features like message transformations are often not suitable due to the large payloads sizes in scientific calculations. At the moment the WSO2 ESB [10] is used at IPP Greifswald. The ESB is an Open Source solution and can therefore be extended in various ways. Such an extension was implemented in the form of a new load balancing module for long running web service calculations at W7-X. The module performs a distribution based on the number of active connections to the service endpoints.

\section{MHD web services for W7-X}

In the last years a growing number of web services were designed, implemented and operated at IPP Greifswald. This includes physics model services for MHD calculations as well as diagnostic modeling (see Fig. 4). The results of web service calculations and other often-needed information are stored in databases which are also accessible via web services interfaces.

\section{VMEC web service}

The Variational Moments Equilibrium Code (VMEC) [4] solves the magnetohydrodynamic force balance in a three-dimensional geometry. The code is commonly used in the stellarator community, e. g. at LHD [11]. VMEC data can be used for flux surface mapping for the analysis of data from profile diagnostics (Thomson scattering, ECE, X-ray spectrometers) and is often the basis for further modeling like stability, transport, plasma edge behavior and other codes.

The VMEC computation is based on the assumption of closed nested flux surfaces and therefore does not include magnetic islands or stochastic regions. The code uses a curvilinear coordinate system with the normalized toroidal flux $s$ as the radial coordinate and two angels for the toroidal and poloidal direction. The $s$ coordinate represents a flux surface label with values from 0 (magnetic axis) to 1 (last closed magnetic surface).

For free-boundary calculations, the code uses the vacuum magnetic field by external coils, pressure- and toroidal current profiles, the total toroidal flux, and an initial guess for the position of magnetic axis and plasma boundary. For the calculation of a consistent equilibrium, the user has to provide the coil geometry and currents for the vacuum magnetic field. The pressure and toroidal current profile can be provided from data of profile diagnostics (Te and ne), magnetic diagnostics [12], and from models like transport codes, heating (ECRH, NBI) and current drive. For the initial guess for magnetic axis and boundary a suitable vacuum configuration or data from function parameter-ization can be used. Dependent on the input conditions and chosen grid resolution, a VMEC calculation can take several minutes to compute.

The VMEC web service provides a SOAP/WSDL and a REST interface. As the original VMEC is written in FORTRAN, the web service acts as a Java wrapper which calls the VMEC executable. For the execution of VMEC calculations, the web service uses an asynchronous request pattern. The user starts a calculation by providing the input parameters in form of a conventional VMEC input file or an input object and receives an identifier. This ID can be used for status checks and later for requesting the output in different forms. The input and output data of a calculation are stored on the server and can be recovered at any time. The web service interface allows also the usage of the original input and output files of VMEC. This is beneficial for the usage of web service data by the numerous codes working with VMEC output files. The web service also provides functions for the access to frequently requested quantities. This can be done by using a calculation ID and includes values like the position of the magnetic flux surfaces or the rotational transform (iota [13]) profile. Additional features are the 
transformation from VMEC coordinates to other coordinate systems, e.g. Cartesian or Cylindrical coordinates and conversions from $s$ to the effective plasma radius $\left(\mathrm{r}_{\text {eff }}\right)$.

The WSDL interface was created using the Contract-first approach, aiming for data types and functions signatures in alignment to other W7-X web services and the Minerva data analysis framework [6]. For example the class magnetic configuration is used for coil and current definitions. This class is designed as a general container for all kinds of elements that generate a field. The VMEC service also uses the same definitions for Fourier coefficients and other common data types like vectors or coordinates.

The Minerva framework uses the VMEC web service for the calculation of 3D MHD equilibrium data. The framework reads data from existing calculations or creates new equilibrium data based on the given parameters. For this purpose Minerva uses the input parameters for the creation of a hash value, which is used as the calculation ID of a corresponding VMEC equilibrium. This leads to an automatic creation of suited VMEC equilibrium data within the execution of the Bayesian model of Minerva.

\section{Further physics model services}

Next to the VMEC service, additional MHD model web services were implemented: MAG3D, Extender, and Field Line Tracer are examples which are currently in use at W7-X. Travis and a helium beam web service are examples for diagnostic modeling.

MAG3D [3] provides functions for the calculation of magnetic fields and vector potentials using Biot Savart's law. The service provides a SOAP/WSDL interface. The MAG3D interface introduces the magnetic configuration object, which is also used in the Minerva framework and in other W7-X web services. The web service is used for the creation of so called Mgrid files for VMEC. These files contain vacuum magnetic field information which is used for free-boundary calculations of VMEC.

The W7-X FP web service is a Java reimplementation of a Function Parameterization code for W7-X configurations [14]. It provides a fast recovery of equilibrium parameters based on a fixed number of input parameters: coil currents, minor radius, pressure profile and plasma current profile. The code provides an interpolation based on a large number of pre-calculated equilibrium calculations, which represent the parameter space of W7-X. In this way the web service can provide a fast assumption of the iota profile, magnetic coordinates and flux surfaces position within several milliseconds. Although it does not perform a real equilibrium calculation, the FP information is accurate enough for many online analysis and plasma operation use cases. VMEC can be used if a full MHD equilibrium calculation is needed (e.g. for stability- or transport simulations), but it also doesn't cover the plasma boundary.

The Extender [15] code calculates the magnetic field and vector potential outside of the core plasma, based on MHD equilibrium data from different 3D codes including VMEC. It uses a virtual casing principle for the computation of the magnetic field from external coils and plasma currents. The Extender code is implemented in $\mathrm{C}++$ and uses parallelization via Message Passing Interface (MPI). The web service runs therefore on a virtual machine with multiple cores. The Web service is a Java wrapper providing a SOAP/WSDL interface. The interface uses the same data types as VMEC and MAG3D e.g. for the definition of vectors or the magnetic configuration. The Extender web service also supports the usage of calculation IDs from the VMEC web service.

Field Line Tracer [16] is a SOAP/WSDL web service for field line tracing and the calculation of related quantities. Therefore it is able to perform different tasks like the calculation of connection lengths, Poincarè maps, or diffusion simulations incl. estimations of heat fluxes to plasma-facing components. The service can use different configurations of $\mathrm{W} 7-\mathrm{X}$ as well as other machines: provided as coil data for vacuum configurations or as magnetic field values on a grid e. g. for equilibrium calculations. As field line tracing is an elementary feature, the service is used for many calculations at IPP Greifswald. Service instances run on several virtual machines. Additional functions were added to improve the usability of the web service. This includes a new website with documentation of the service functions and data types as well as an interactive monitoring which shows the status of the service endpoints. 
Travis [17] is a ray-tracing code for electron cyclotron simulations, e.g. for ECRH operation scenarios, ECE and Reflectometry diagnostic. The Travis web service provides a SOAP/WSDL interface as well as status information on a website. The service uses MHD equilibrium data, which can be provided by a link from the VMEC web service.

Another example for diagnostic modeling is a web service for simulations of a thermal helium beam [17]. The service is a Bayesian based Monte Carlo simulation for a collisional-radiative model. For a typical simulation run the service calls are distributed to several endpoints on virtual machines (typical 10 to 20). The service is implemented in C\# as a SOAP/WSDL web service.

\section{Databases and repositories}

Databases are used to store engineering and physics model data, as well as diagnostic and operational data of W7-X experiments.

CoilsDB [16] is a database for magnetic coil data. The database can be accessed by REST or SOAP/WSDL interface. CoilsDB is implemented as an object-oriented database written in Python and stores coil geometry data and currents, as well as metadata. At the moment the database hosts more than 1200 single coils and multiple coil configurations, which is a full set of all magnetic coils of a machine and the corresponding coil currents. This includes W7-X data as well as data of other machines (Fig. 5). The magnetic system of W7-X comprises 70 superconducting coils: 10 coils of 7 different types. Coil geometries can be found in as-designed and as-built states including results of the finite element modeling of a coil shape deformation under electromagnetic loads [21]. In addition there are 10 control and 5 trim coils.

ComponentsDB [16] is a database which contains 3D model data of machine components like the W7$X$ plasma vessel, diagnostic ports, heat shields, or divertor units. Many models were converted from the CAD data of W7-X machine components. The model data are meshed representations in a resolution which is suitable for physics calculations. The database is implemented in Python and can be accessed via a REST or SOAP/WSDL interface. Related functions for the creation of new mesh data or intersection tests are also available via web service. The Field Line Tracer supports the usage of data from ComponentsDB and CoilsDB by database ID.

The ArchiveDB Web API [22] is a RESTful web service for the W7-X archive. The archive is the central database for the experiment data of W7-X. This includes raw and analyzed data of the diagnostic systems as well as operational data of the W7-X sub-systems like vacuum systems or cryo supply. The web service provides read access to all data in a unified form and it can also be used to write analyzed data to the archive.

The W7-X Reference Equilibria is a collection of selected VMEC calculations for W7-X experiments. It is accessible via the SOAP/WSDL and REST interfaces of the VMEC web service. The collection contains calculations for the first operational phase as well as for the basic configuration variants and optimized configurations [23] of W7-X. All reference calculations can be accessed by a unique ID. The REST interface includes a listing of all available reference equilibria and a preview website with interactive plots (flux surfaces, iota and pressure profile) and other features. The preview webpage contains a description of the corresponding equilibrium and displays characteristic values like major/minor radius, beta, and the average magnetic field on the axis (see Fig. 6).

\section{Conclusion and Outlook}

Web services are a well suited way to exposure heterogeneous software to a wider audience. Standard interfaces with aligned data types allow a flexible combination of components on an application level. Several web services were successfully implemented in the last years at IPP Greifswald. The services provide function calls to physics model codes as well as access to data from repositories and databases. A web service for the 3D equilibrium code VMEC was implemented: accessible from user code in multiple programming languages or from an integrated data analysis framework. The operation of web 
services is strongly supported by SOA infrastructure in form of an Enterprise Service Bus and a central information website. Status monitoring for long running calculations was implemented for several services. The services run on virtual machines in a private Cloud. It is planned to upgrade the hardware of this system continuously to meet the growing user demand. Further plans include the expansion of existing service features and the implementation of new services. For example functions for searching and filtering of data from the VMEC web service. Such features become more and more important with a growing number of equilibria. In addition, users are interested in further model data which could also be provided as web services e.g. for a database of (neoclassical) transport coefficients.

\section{Acknowledgement}

This work has been carried out within the framework of the EUROfusion Consortium and has received funding from the Euratom research and training programme 2014-2018 under grant agreement No 633053. The views and opinions expressed herein do not necessarily reflect those of the European Commission.

\section{References}

[1] H.-S. Bosch, R. Brakel, M. Gasparotto et al., "Transition from Construction to Operation Phase of the Wendelstein 7-X Stellarator", IEEE Trans. on Plasma Science., vol. 42, no. 6 (2014), pp. 432-438

[2] A. Werner, et al. "Integrated Software Development for Wendelstein 7-X" FT/P7-6, 21st IAEA Fusion Energy Conference, Chengdu, 2006.

[3] J. Svensson and A. Werner "Large Scale Bayesian Data Analysis for Nuclear Fusion Experiments", Proceedings IEEE Workshop on Intelligent Signal Processing WISP 2007

[4] S. P. Hirshman and W. I. van Rij "Three-dimensional free boundary calculations using a spectral Green's function method" Computer Physics Communications 43 (1986) 143-155

[5] A. Werner, et al "Scientific component framework for W7-X using service oriented GRID middleware" Fusion Eng. and Des. 85 (2010) 394-398

[6] J. Svensson, A. Dinklage, R Fischer, J. Geiger, A. Werner, "Integrating Diagnostic Data Analysis for W7-AS using Bayesian Graphical Models", Rev Sci Instrum, vol. 75, pp. 4219-4221, 2004

[7] World Wide Web Consortium: https://www.w3.org/

[8] R.T. Fielding "Architectural Styles and the Design of Network-Based Software Architectures", Ph.D Thesis, University of California (2000)

[9] VMware vSphere: http://www.vmware.com/products/vsphere.html

[10] WSO2 ESB: http://wso2.com/products/enterprise-service-bus/

[11] S. A. Lazerson, S. Sakakibara and Y. Suzuki “A magnetic diagnostic code for 3D fusion equilibria”, Plasma Phys. Control. Fusion 55 (2013) 025014 (8pp)

[12] M. Endler, et al. "Engineering design for the magnetic diagnostics of Wendelstein 7-X", Fusion Eng. Des., 100 (2015) 468-494

[13] A.H. Boozer "Physics of magnetically confined plasmas", Rev. Mod. Phys. 76 (2004) 1071

[14] A. Sengupta, et al. "Fast recovery of vacuum magnetic configuration of the W7-X stellarator using function parametrization and artificial neural networks", Nucl. Fusion, 44 (2004) 1176

[15] M. Drevlak, D. Monticello and A. Reiman "PIES free boundary stellarator equilibria with improved initial conditions", Nuclear Fusion, 45 (2005), 731

[16] S. A. Bozhenkov, et al "Service oriented architecture for scientific analysis at W7-X. An example of a field line tracer" Fusion Eng. Des., 88 (2013) 2997-3006

[17] N. B. Marushchenko, Y. Turkin, and H. Maaßberg, "Ray-tracing code TRAVIS for ECR heating, EC current drive and ECE diagnostic", Comput. Phys. Commun. 185, 165 (2014). https://doi.org/10.1016/j.cpc.2013.09.002

[18] M. Krychowiak, et al. "Bayesian modelling of a thermal helium beam for measurement of electron density and temperature in the W7-X divertor plasma", Plasma Phys. Contr. Fusion 53 035019, https://doi.org/10.1088/0741$\underline{3335 / 53 / 3 / 035019}$

[19] M. Otte, et al. "The WEGA stellarator: Results and prospects", In Plasma 2007 - Proceedings Intl. Conf. on Research and Applications of Plasmas: 4th German-Polish Conference on Plasma Diagnostics for Fusion and Applications. Vol. 993. 2008. p. 3-10

[20] F. Schauer, K. Egorov and V. Bykov "HELIAS 5-B magnet system structure and maintenance concept", Fusion Eng. Des., 88 (2013), 1619

[21] T. Andreeva, T. Bräuer, V. Bykov, K. Egorov, M. Endler, J. Fellinger, J. Kißlinger, M. Köppen and F. Schauer "Tracking of the magnet system geometry during Wendelstein 7-X construction to achieve the designed magnetic field", Nucl. 
Fusion, 55 (2015), 063025

[22] M. Grahl, et al. "Archive WEB API: A web service for the experiment data archive of Wendelstein 7-X", Fusion Eng. Des. (2017), https://doi.org/10.1016/j.fusengdes.2017.02.047

[23] J. Geiger, et al. "Physics in the magnetic configuration space of W7-X", Plasma Phys. Control. Fusion, 57 (2014), 014004 


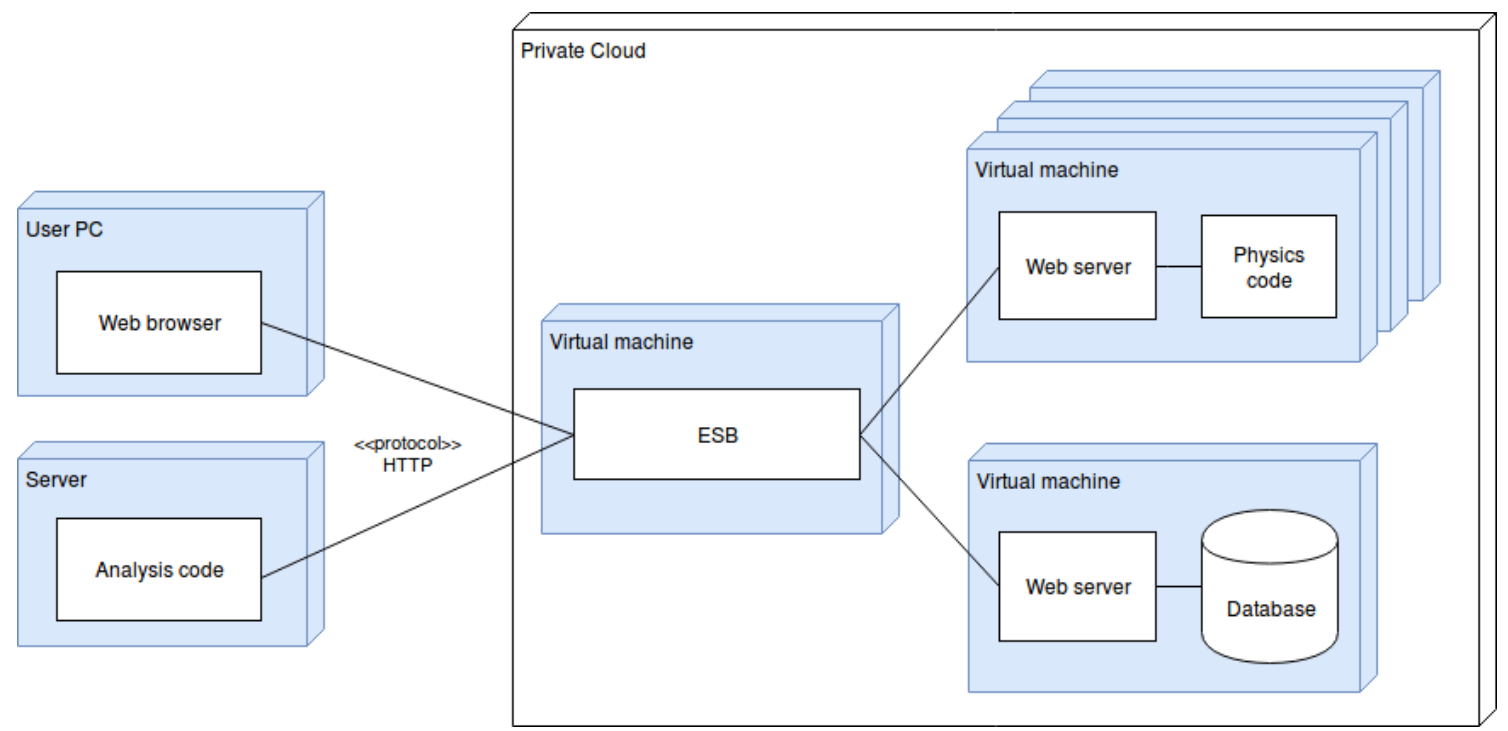

Fig. 1 Deployment view of W7-X web services. Web services run on virtual machines in a private Cloud environment. In most cases an implementation of a SOAP/WSDL interface or REST API runs on a standard web server and calls an existing code or database. An Enterprise Service Bus (ESB) is used for endpoint abstraction: a proxy endpoint can represent several instances of the same service while the ESB distributes the incoming requests to the original endpoints for load balancing.

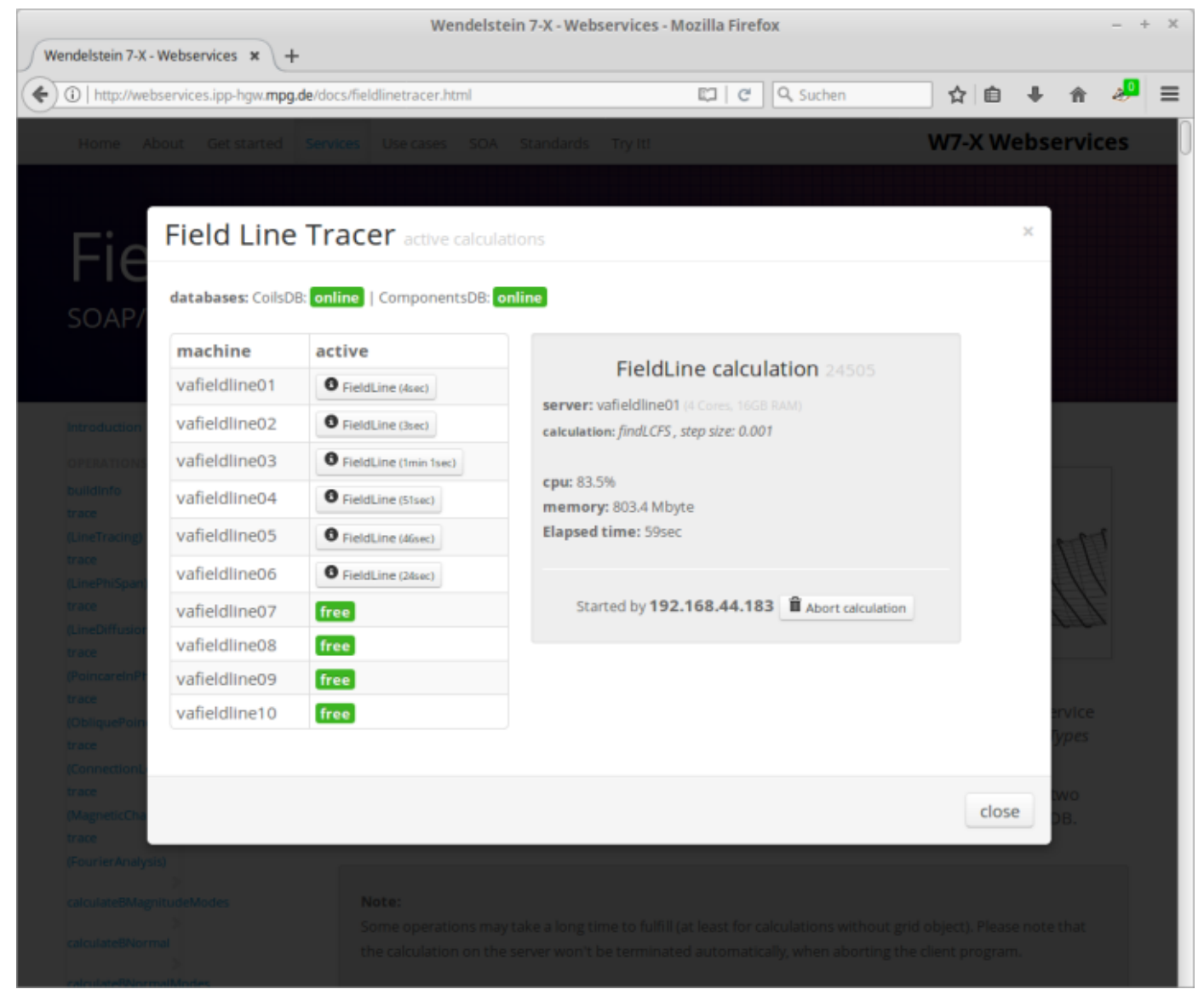

Fig. 2 Online monitoring for long running web service calculations. 


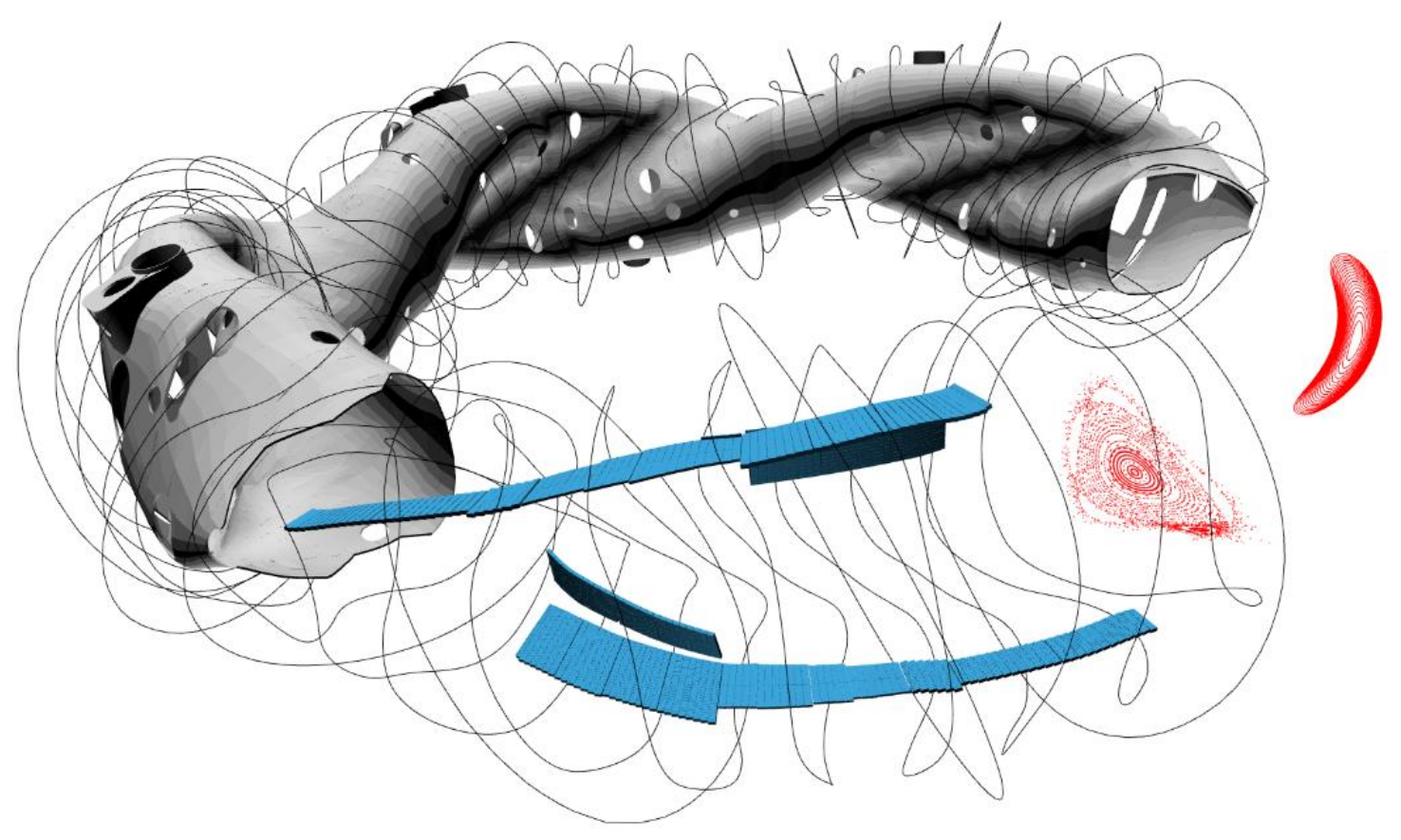

Fig. 3 Visualization of data from W7-X web services in 3D. Planar and non-planar magnetic coils from CoilsDB are shown in black. Examples of the data from ComponentsDB: divertor units of one module in blue and three modules of the W7-X plasma vessel including diagnostic ports in gray. The flux surface plot at phi $=0^{\circ}$, obtained from equilibrium data from the VMEC web service, as well as a Poincarè plot (triangle-shaped plane with islands) coming from Field Line Tracer, are represented in red. The data is displayed in a web browser using WebGL, as part of an interactive application. The user can select data from W7-X web services, rotate the camera, zoom in, etc.

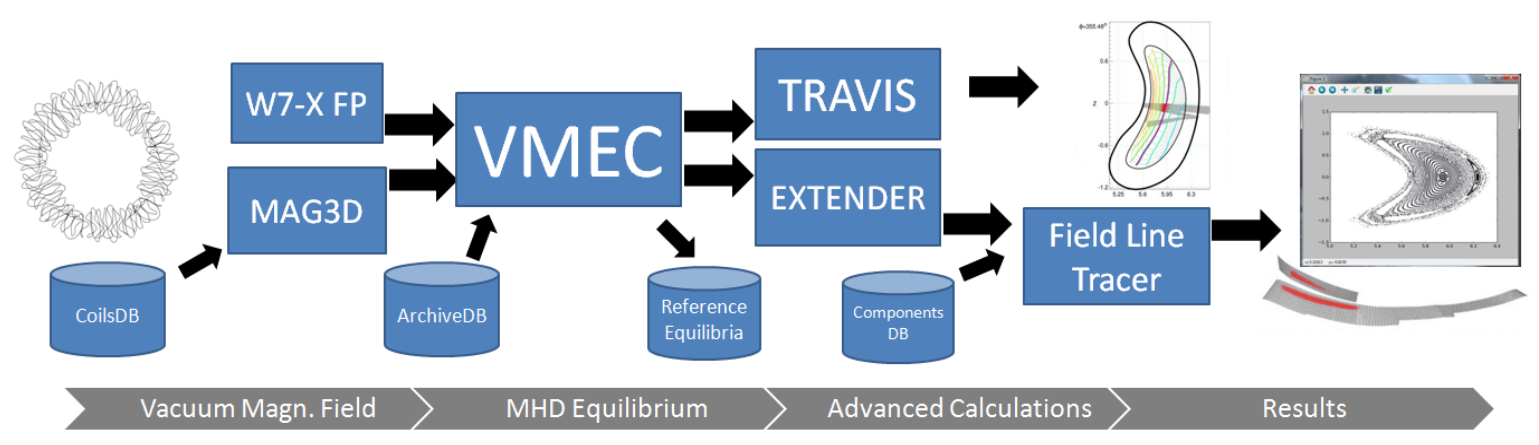

Fig. 4 Orchestration of W7-X web services. 


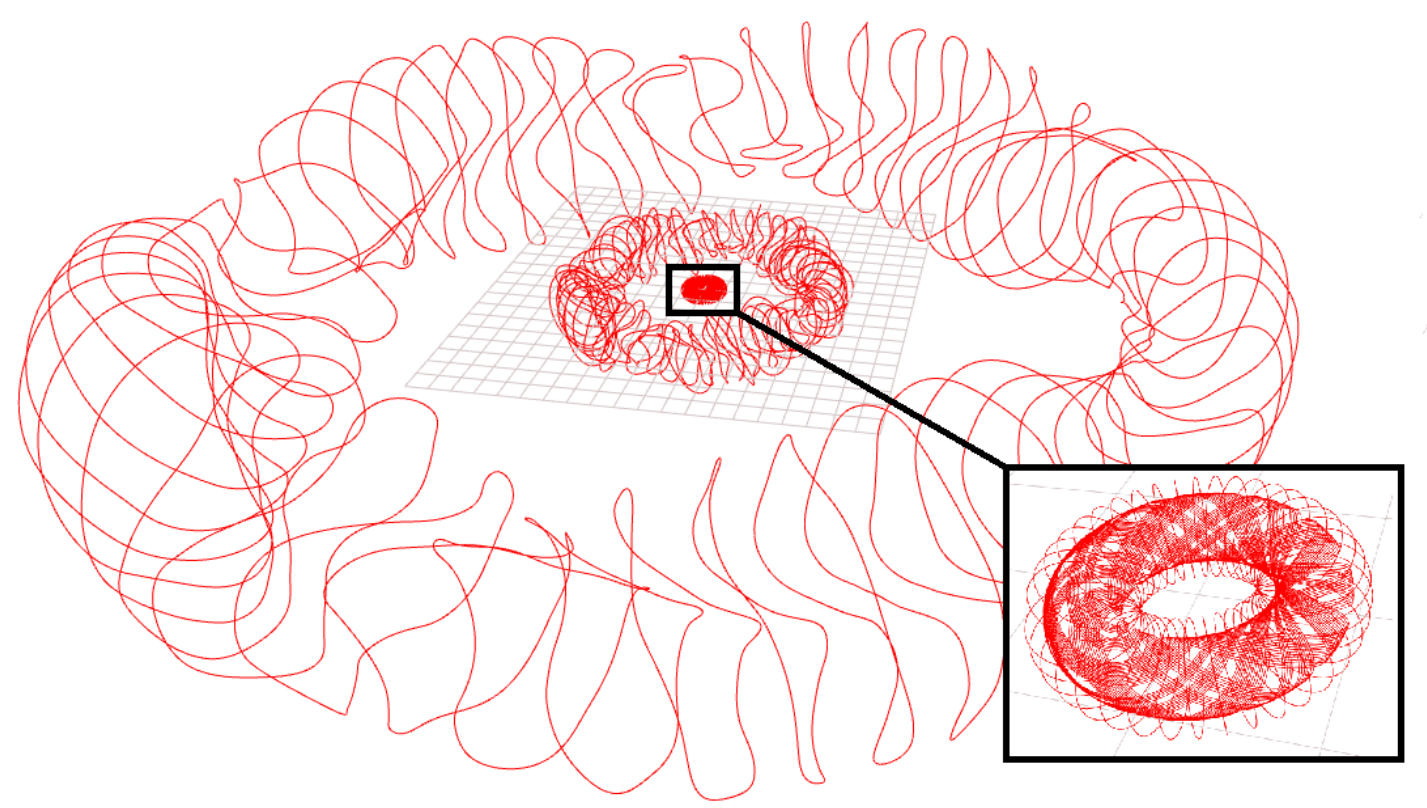

Fig. 5 Examples of CoilsDB data from different machines: WEGA (major radius of $72 \mathrm{~cm}$ ) [19], Wendelstein 7-X $(\mathrm{R}=5.5 \mathrm{~m})$, and the reactor study HELIAS 5-B $(\mathrm{R}=22 \mathrm{~m})[20]$.

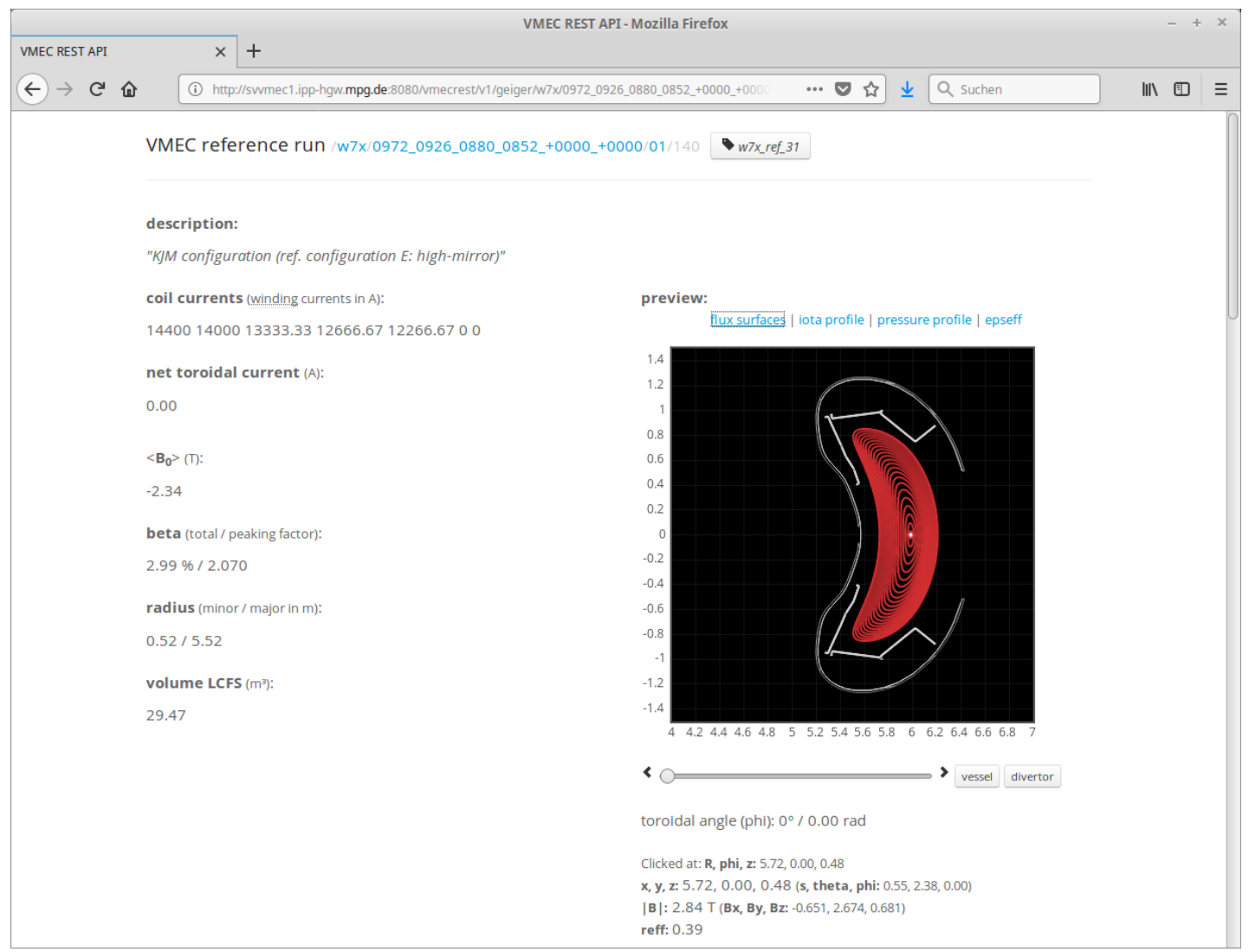

Fig. 6 Online preview page. A website shows the status and main parameters (e.g. beta, coil currents, iota profile) of VMEC calculations. This includes an interactive flux surface plot. By clicking on a point within the plot, the user gets the position in different coordinate systems as well as the magnetic field and reff value. The preview pages are generated on-the-fly for calculations coming from the VMEC web service (incl. W7-X Reference Equilibria). 